\title{
Physics of magnetic confinement fusion
}

\author{
F. WAGNER \\ Max-Planck-Institut für Plasmaphysik - Greifswald, Germany, EURATOM Association \\ and Research Laboratory for Advanced Tokamak Physics \\ St. Petersburg Polytechnic State University - St. Petersburg, Russia
}

\begin{abstract}
Summary. - Fusion is the energy source of the universe. The local conditions in the core of the Sun allow the transfer of mass into energy, which is finally released in the form of radiation. Technical fusion melts deuterons and tritons to helium releasing large amounts of energy per fusion process. Because of the conditions for fusion, which will be deduced, the fusion fuel is in the plasma state. Here we report on the confinement of fusion plasmas by magnetic fields. Different confinement concepts - tokamaks and stellarators - will be introduced and described. The first fusion reactor, ITER, and the most modern stellarator, Wendelstein 7-X, are under construction. Their basic features and objectives will be presented.
\end{abstract}

\section{1. - Introduction}

Energy is the most important commodity man needs on Earth. It provides mankind with food and energy makes it largely independent of the perils of nature. It is not astonishing that energy consumption rose in the last centuries in parallel to the number of inhabitants on Earth. The relation between consumed energy and population is about quadratic because the per-capita energy consumption also increases over the years. It is expected that energy consumption will double till 2050 as a consequence of the population 
rise from presently 7 billion to about 9 billion people. Such a rise in consumption would be even steeper than quadratic.

On the other hand, the availability of affordable energy allowed mankind to grow. Whereas our metabolism would require about $100 \mathrm{~W}$ the power an individual actually "consumes" e.g. in the highly developed OECD countries is between 4 and $5 \mathrm{~kW}$. In this case each representative has about 40-50 "slaves" working continuously on his or her behalf. The basis of this wealth are the fossil fuels, which mankind has learned to effectively exploit and to apply in an unthinkable treasure of technologies. The consequences of this growth are twofold: 1) After a fairly short period in the history of mankind, the fossil fuels will be exploited; 2) their chemical nature causes the $\mathrm{CO}_{2}$ concentration of the atmosphere to rise with a change in the Earth radiation balance as a corollary. The primary consequence of this evolution is that new and $\mathrm{CO}_{2}$-free energy sources have to be developed. There are only three possibilities: renewable energies in their different occurrences, fission based on breeder technology and fusion.

Fusion represents a fundamental process in nature. The primary energy source of the universe is based on fusion processes. Fusion provided the cosmos with energy since 13.8 billion years. Developed on Earth, fusion would represent a clean and basically inexhaustible energy source. Worldwide, science and research are engaged in the development of this technology. In this chapter, we describe the fundamental principles of fusion and the status and prospects of fusion $\mathrm{R} \& \mathrm{D}$ on the basis of magnetic confinement. An easily readable introduction into all aspects of fusion for a not necessarily technical readership is ref. [1]; there are many excellent text books on high-temperature plasma physics and fusion technology, I would recommend ref. [2]. A more detailed version of this paper is given in ref. [3].

\section{2. - The role of fusion in the universe}

The big-bang left leptons along with protons, deuterons, a little helium and spurious traces of lithium. The rest of the 92 elements up to uranium are the spin-offs of the self-organisation of the universe enforced by gravity - the formation of galaxies with stars consisting of hydrogen acting as breeder material for new elements. In the core of the Sun the temperature is $15 \mathrm{MK}\left(\sim 1.5 \mathrm{keV}\left({ }^{1}\right)\right)$ and the pressure is $10^{16} \mathrm{~Pa}$. Under these extreme conditions matter is in the fourth state - the plasma state. Like in the other cases — solid, liquid, gas - the new state emerges out of the previous one from a disintegration process through elevated temperatures: In the transition from gas to plasma the constituents - molecules and atoms - are dissociated or ionized, respectively, and free electrons and ions are produced - e.g. protons in case of hydrogen as working gas. Though we are initially describing a process of disintegration, at the end, new elements are formed during the life-cycle of stars by fusion of light elements to heavier ones.

$\left.{ }^{1}\right)$ In plasma physics, temperatures are expressed by eV and keV. $1 \mathrm{eV}$ corresponds to $11600 \mathrm{~K}$. 
The initial fusion process is the fusion of hydrogen to helium. The most basic process is the pp I cycle $\left({ }^{2}\right)$. In a complex step process, 4 protons $\left({ }^{1} \mathrm{H}\right)$ yield helium $\left({ }^{4} \mathrm{He}\right)$. The individual steps are

$$
\begin{aligned}
& { }^{1} \mathrm{H}+{ }^{1} \mathrm{H} \rightarrow \mathrm{D}+\mathrm{e}^{+}+\nu_{\mathrm{e}}+0.42 \mathrm{MeV}, \\
& \mathrm{D}+{ }^{1} \mathrm{H} \rightarrow{ }^{3} \mathrm{He}+5.49 \mathrm{MeV}, \\
& { }^{3} \mathrm{He}+{ }^{3} \mathrm{He} \rightarrow{ }^{4} \mathrm{He}+2{ }^{1} \mathrm{H}+12.86 \mathrm{MeV} .
\end{aligned}
$$

The transformation of a proton into a neutron in the initial step (1) is governed by the weak interaction and is, therefore, an extremely slow process. This is essential for the lifetime of the Sun and our planetary system. Besides a more massive element, He, a positron, $\mathrm{e}^{+}$, and a neutrino, $\nu_{\mathrm{e}}$, are additionally created, which share the released energy. The total energy gained in this process is $26.72 \mathrm{MeV}$. This is the sum of the exothermal processes (1) and (2) taken twice and the process (3). In process (1) the annihilation $\gamma$-energy of $\mathrm{e}^{+}$with an abundant electron $(1.02 \mathrm{MeV})$ is included in the energy balance (because the $\gamma$-radiation adds to the fusion pressure to be balanced by Sun's gravity). Less than $1 \%$ of the masses involved are transferred into energy.

Fusion processes yielding higher mass elements are exothermal releasing vast amounts of energy. The energy process behind fusion is the transformation of mass into energy according to Einstein's most famous relation $E=m c^{2}$, which is one of the consequences from the special theory of relativity published in 1905 .

The burning of protons to helium will continue in the Sun till the abundance of the ash, helium, will stop this process in the core. In this long-lasting process over billions of years the Sun exists in a state of equilibrium. Force balance is established between gravitation, pointing to the core, and pressure pointing with its (negative) gradient to the edge. The pressure gradient is maintained by the fusion power produced in the core and radiated off at the edge. In between, energy is transported first by radiation then by convection. This balanced equilibrium is lost in the solar life cycle when the hydrogen burn terminates in the core but continues in the outer sphere. Then the Sun grows to a red giant - a short period in its life leaving back the Earth as a rock with a polished surface.

In stars larger than the Sun, the He core continues with fusion utilizing the availability of He as new building brick. But the next element, ${ }^{8} \mathrm{Be}$ is not stable. The triple-alphaprocess $3{ }^{4} \mathrm{He} \rightarrow{ }^{12} \mathrm{C},{ }^{12} \mathrm{C}$ being stable itself, involves three particles and has, therefore, a low probability. At rescue comes that the intermediate step, $2^{4} \mathrm{He} \rightarrow{ }^{8} \mathrm{Be}$, is enhanced because of a fortuitous energy resonance between the two $\alpha$-particles and a low excitation energy level of the ${ }^{8} \mathrm{Be}$ nucleus. In this fusion process the rate is high because also the final process to ${ }^{12} \mathrm{C}$ is energetically enhanced by a nuclear resonance. In this way the probability for the otherwise low-probability 3-body process from ${ }^{4} \mathrm{He}$ to ${ }^{12} \mathrm{C}$ is strongly

$\left({ }^{2}\right)$ There are several chain processes starting from hydrogen and ending in helium. Under solar conditions, the pp I cycle is the most dominant one producing $86 \%$ of the He. The CNO cycle is dominant in stars hotter than the Sun. 


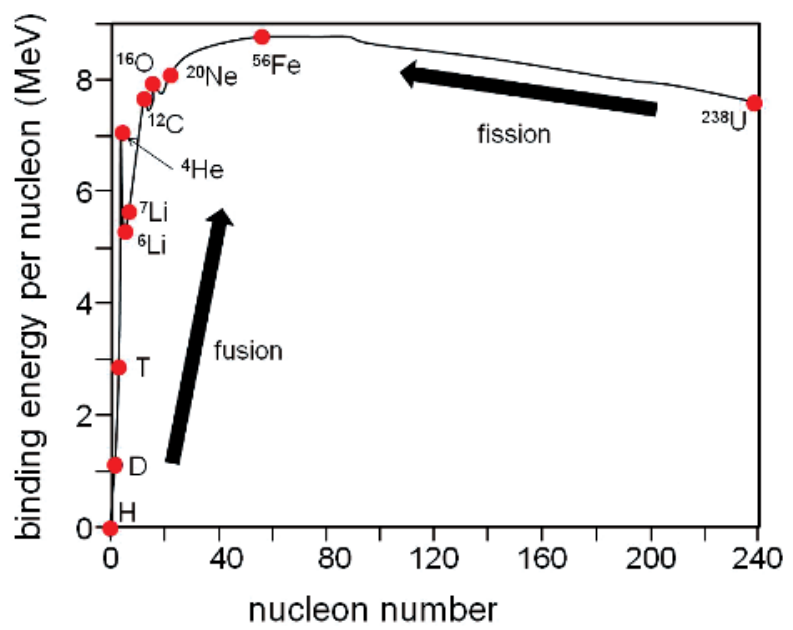

Fig. 1. - The binding energy per nucleon against the nucleon number. The fusion and fission branches are indicated as well as the locations of the elements, which play a role in this paper.

increased. These resonances were brilliantly concluded by Fred Hoyle from the cosmic abundance of ${ }^{12} \mathrm{C}$ (similar with ${ }^{16} \mathrm{O}$ and ${ }^{20} \mathrm{Ne}$ ) before they were experimentally verified.

In more massive stars the fusion processes continue involving $\alpha$-reactions. In this way, ${ }^{16} \mathrm{O}$ forms out of ${ }^{12} \mathrm{C},{ }^{20} \mathrm{Ne}$ from ${ }^{16} \mathrm{O}$ and so on. These processes continue ultimately up to ${ }^{56} \mathrm{Fe}$. Then the means of the fusion process are exploited. This development toward iron is governed by the nuclear binding energy $E(Z, N)=\Delta M(Z, N) \cdot c^{2}$ of a nucleus with mass $M(Z, N)$ with $Z$ protons and $N$ neutrons, which is given by the mass defect $\Delta M(Z, N)=\left[Z \cdot M_{\mathrm{p}}+N \cdot M_{n}-M(Z, N)\right]\left({ }^{3}\right)$. In case of ${ }^{4} \mathrm{He}, E=28.3 \mathrm{MeV}$ or $7.1 \mathrm{MeV}$ per nucleon. Figure 1 shows the binding energy per nucleon as a function of the atomic mass number. The exceptional situation of ${ }^{4} \mathrm{He}$ is shown. Fusion processes ending in He show the largest exothermal response; the burning of ${ }^{1} \mathrm{H}$ to ${ }^{4} \mathrm{He}$ releases most of the energy available to fusion. The maximum of the binding energy is with ${ }^{56} \mathrm{Fe}$.

The branch toward higher mass elements has a different formation history utilizing the strong neutron fluxes emerging in supernovae. With fission, mankind has learned to utilize the energetic possibilities of the branch beyond the binding-energy maximum. About 450 fission reactors are in operation worldwide. A successful demonstration of technical fusion is, however, still missing. With the ITER fusion project, presently under construction in France (see sect. $\mathbf{9}^{*}$ ), the scientific and technical viability of fusion will be demonstrated within the next 10-15 years.

The fusion process between positively charged nuclei has to overcome the Coulomb repulsion. The potential energy at a distance of the proton diameter is about $0.6 \mathrm{MeV}$.

$\left({ }^{3}\right)$ The binding energy is always a positive quantity. 


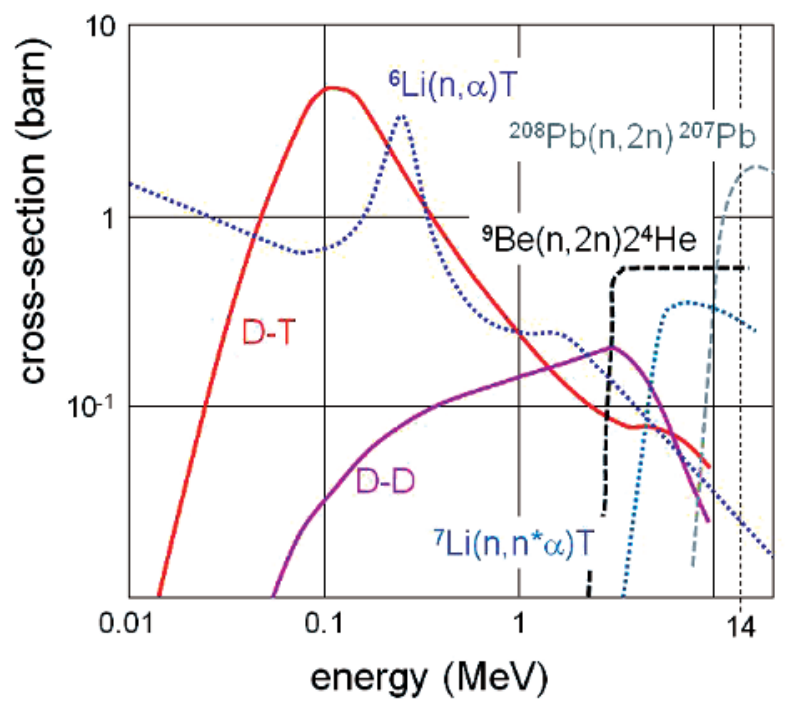

Fig. 2. - Fusion relevant cross-sections are plotted against energy. In case of the fusion reactions, $\mathrm{D}-\mathrm{T}$ and $\mathrm{D}-\mathrm{D}$, the energy is the kinetic energy of the incident particle; in case of the Li-breeding processes and the $(\mathrm{n}, 2 \mathrm{n})$ processes it is the one of the incident neutron.

But nature eases fusion because the particle, the proton, adopts in this interaction the characteristics of a wave, which allows it to leak into the energetically prohibited zone and to finally tunnel through the Coulomb barrier into the deep bound states of the newly formed nucleus. The potential energy at the distance of the de Broglie wave length is about two orders of magnitude lower than the one within the range of the nuclear forces.

The fusion yield depends on the density of the particles, their relative velocity and the nuclear interaction in the form of the fusion cross-section $\sigma_{\text {fus }}$. Fusion is only one form of interaction in a plasma, even the one with the smallest cross-section. The competing interaction processes are Coulomb collisions between charged particles. As the Coulomb collision rate is large compared to the fusion rate the proton assembly (as well as the one of the electrons) can be described by a Maxwellian distribution. The governing kinetic parameter is the temperature rather than the individual particle energy in the assembly interactions - scattering and fusion. This is the reason why we are speaking of controlled thermo-nuclear fusion.

\section{3. - Processes for technical fusion}

Fusion scientists are trying to realize a technology, which basically transfers mass into energy. (Of course, this is again only a transformation process; energy is conserved when mass is transferred e.g. into radiation.) A gram of matter transferred into energy e.g. via processes (1) to (3) would yield $10 \mathrm{GWh}$. This energy would serve the members of a typical OECD family throughout their life. However, technical fusion cannot resort to the 
process acting in the interior of the Sun. A reaction has to be selected, which maintains the number of protons and neutrons circumventing the weak interaction. There are many such processes with different interesting features like the avoidance of free neutrons and thus the avoidance of neutron-induced radioactive fusion waste. The selection of the proper reaction is guided, however, by searching for the highest reaction rate providing the highest fusion yield. This condition is fulfilled by fusing deuterium $\mathrm{D}={ }^{2} \mathrm{H}$, the heavy isotope of hydrogen, with tritium $\mathrm{T}={ }^{3} \mathrm{H}$, the super-heavy one. The reaction reads

$$
\mathrm{D}+\mathrm{T} \rightarrow{ }^{4} \mathrm{He}(3.52 \mathrm{MeV})+n(14.06 \mathrm{MeV}) .
$$

The fusion energy per process $E_{\text {fus }}=E_{\alpha}+E_{\mathrm{n}}=17.58 \mathrm{MeV}$. The DT fusion cross-section is plotted against energy in fig. 2 and compared with the alternative DD fusion process. DT fusion has the higher cross-section and the maximum is at lower energy: larger fusion yield is possible at less demanding conditions. The basic reaction (4) highlights already the major characteristics of fusion energy: There are no radioactive process products and no $\mathrm{CO}_{2}$ release. Deuterium can be gained from seawater. The DT reaction is, however, not neutron-free. The neutron is actually the dominant energy carrier. It activates structural material and fusion energy leaves radioactive waste, which, however, quickly decays and is characterised by a comparatively short-lived radiotoxicity.

The reaction products, $\alpha$-particle and neutron, have additional tasks. The $\alpha$-particle provides the necessary inner heating of the plasma to maintain the conditions for fusion; the fusion neutron allows producing tritium via a breeding reaction with lithium.

Tritium is a radioactive substance with a short half-life of 12.3 years. Therefore, it is naturally not available in any relevant concentration rather a technology has to be developed for its production. From reaction (4) the annual tritium use of a fusion power station with $3 \mathrm{GW}_{\text {th }}$ power can be calculated to be - in an idealized case - $167 \mathrm{~kg}$. The breeding technology of tritium is based on the nuclear interaction of a neutron with lithium ( $\mathrm{Li}$ ), which yields tritium. There are two (stable) $\mathrm{Li}$ isotopes of relevance, ${ }^{6} \mathrm{Li}$ and ${ }^{7} \mathrm{Li}$. Natural lithium contains $7.5 \%{ }^{6} \mathrm{Li}$ and $92.5 \%{ }^{7} \mathrm{Li}$. The breeding reactions are

$$
n+{ }^{6} \mathrm{Li} \rightarrow \mathrm{T}+{ }^{4} \mathrm{He}+4.8 \mathrm{MeV}
$$

This process, involving a thermal neutron, is exothermic and represents additionally a substantial contribution to the overall fusion energy yield.

$$
n+{ }^{7} \mathrm{Li} \rightarrow \mathrm{T}+{ }^{4} \mathrm{He}+n^{*}-2.5 \mathrm{MeV}
$$

This process with fast neutrons is endothermic corresponding to a mass difference $\Delta m \sim$ $-0.0027 \mathrm{u}$ whereas $Q=\Delta m^{*} 931.5 \mathrm{MeV}$; the resulting $n^{*}$ has a reduced energy, which is distributed according to the kinetic conditions of its release.

The neutron necessary to initiate the breeding process emerges from the central DT fusion itself starting with an energy of $14.1 \mathrm{MeV}$. The breeding of tritium is a process, 
which happens within the reactor chamber. This is an important safety aspect. In particular, there is no need of external transportation of tritium apart from the initial fuelling of a fusion reactor to start the fusion and breeding processes.

The processes (5) and (6) have greatly different cross-sections - both in magnitude and energy dependence (see fig. 2). The $(n, \alpha)$ cross-section of ${ }^{6} \mathrm{Li}$ has the classical form with a $1 / v$ dependence at low velocities $v$ and a sharp maximum corresponding to a resonance with an energy state of the compound nucleus ${ }^{7} \mathrm{Li}$. The cross-section of the ${ }^{7} \mathrm{Li}$ reaction has the typical shape of a threshold reaction with a fast rise of $\sigma$ beyond a critical energy.

Tritium is produced in a breeding blanket, which surrounds the fusion plasma and absorbs the neutrons from the fusion process. The fusion blanket is a complex compound structure because it has to fulfil three major tasks: 1) produce tritium and allow collecting it, 2) transfer the kinetic energy of the neutrons into a cooling medium and 3) screen the superconducting coils used for magnetic confinement from the central neutron flux to avoid material damage.

\section{4. - The conditions for controlled nuclear fusion}

The plasma parameters relevant for fusion and the fusion conditions proper are obtained from an energy balance. It is easy to formulate the conditions for controlled nuclear fusion but it is difficult to establish them. John Lawson was the first to publish the Lawson Criterion in 1957 [4]. Different formulations exist depending on the terms and processes considered - whether the $\alpha$-particle heating is equated to the power losses (ignition condition), whether the total fusion power is related to the external heating power $P_{\text {ext }}$ (break-even condition), whether part of the fusion power is recycled to provide electricity for the plant operation, whether the ash concentration (He, $\alpha$-particles) is consistently considered or whether radiation losses are included. The outcome of such balances yields average values and can only be considered as rough guidance. The design of a fusion reactor like ITER following the definition of its objectives requires detailed analysis as e.g. summarized in ref. [5].

The power balance for the ignition condition including radiation is

$$
p_{\alpha}=1 / 4 n_{\mathrm{DT}}^{2}\left\langle\sigma_{\mathrm{fus}} v\right\rangle E_{\alpha}=\text { const } n_{\mathrm{e}} n_{Z} Z^{2} \sqrt{ } T+3 / 2\left(n_{\mathrm{DT}} T\right) / \tau_{\mathrm{E}} .
$$

The left-side term represents the $\alpha$-particle power density deposited inside the plasma maintaining the self-sustained burn. The first term on the right side represents radiation $\left.{ }^{4}\right)$, the 2 nd one represents the plasma transport losses parameterized by the energy confinement time $\tau_{\mathrm{E}} \cdot n_{\mathrm{DT}}$ is the deuterium-tritium, $n_{\mathrm{e}}$ the electron and $n_{Z}$ the impurity density (e.g. He or those caused by wall erosion). $T$ is the temperature. The factor $1 / 4$ results from the particle balance: $n_{\mathrm{DT}}=n_{\mathrm{e}}=n_{\mathrm{D}}+n_{\mathrm{T}}$ and an equal mix between $\mathrm{D}$ and $\mathrm{T}$.

$\left({ }^{4}\right)$ In this simplified approach, we assume only Bremsstrahlung-radiation. 


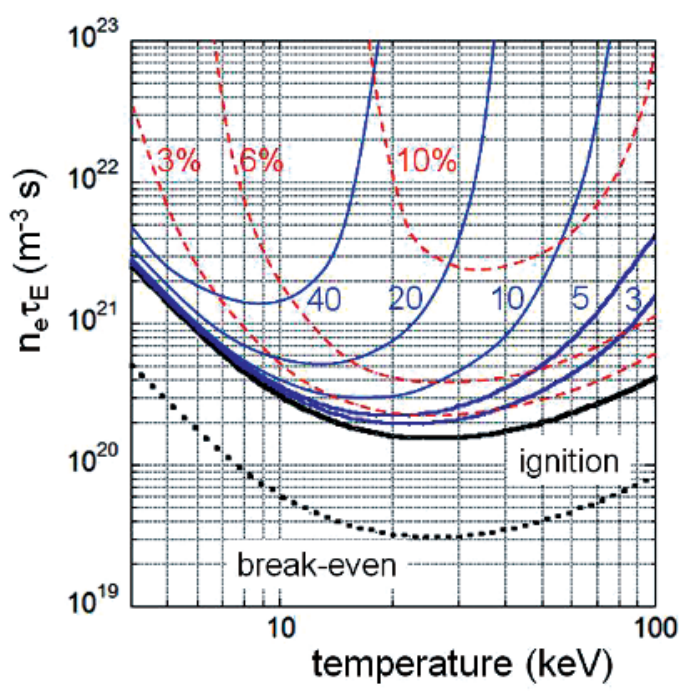

Fig. 3. - The fusion product, electron density $n_{\mathrm{e}}$ times the energy confinement time $\tau_{\mathrm{E}}$, against the plasma temperature. The thick solid line represents the ignition condition, the dotted line the break-even one. The marked curves represent the ignition condition with impurities. The cases for helium are shown by solid lines whereas the respective number is the ratio of particle to energy confinement time $\rho=\tau_{\mathrm{p}} / \tau_{\mathrm{E}}$; the dashed line shows the impact of carbon radiation $(Z=6)$ at different concentrations.

We meet here the term "energy confinement time $\tau_{\mathrm{E}}$ " for the first time though we have used the phrase "magnetic confinement" already. The energy confinement time is the energy replacement time. In case of a house, $\tau_{\mathrm{E}}$ would represent the cool-down time when the stove is turned off. In case of good thermal insulation, $\tau_{\mathrm{E}}$ is long. In case of a plasma $\tau_{\mathrm{E}}$ is determined by plasma transport. The plasma core is heated externally with $P_{\text {ext }}$ or - after ignition - internally with the $\alpha$-particle heating power $P_{\alpha}$. Via heat conduction and convection, this power is transported to the plasma edge and radiated off or deposited onto specific technical installations - limiters or divertor targets. The transport rates are characterized by $\tau_{\mathrm{E}}$, which is a measure of the thermal isolation of the plasma. The global power balance can be written under steady-state conditions as: $\dot{W}=0=P_{\text {ext }}+P_{\text {int }}-W / \tau_{\mathrm{E}}$. For the same heating power, the energy content $W$ doubles if $\tau_{\mathrm{E}}$ doubles. Like in a house a large stove (a large external heating power) is required in case of bad thermal insulation. This is not an economic approach for maintaining a desired temperature. In analogy, as $\tau_{\mathrm{E}}$ is such a crucial parameter for confinement concepts and operational plasma conditions the study of transport and confinement is in the core of fusion research.

Power balance (7) can be casted into the form $n \tau_{\mathrm{E}}=f(T)$. This relation is plotted in fig. 3 for various assumptions - the break-even condition when the fusion output matches the external heat input ("the stove is puffing"), and the ignition conditions 
("the stove transits into self-sustained burn"). The curves have a minimum due to the maximum in the fusion cross-section (see fig. 2). The other cases shown in fig. 3 reflect the role of impurities. An unavoidable "impurity" is He, the fusion ash. Its concentration is determined by a particle balance, which is similarly structured as eq. (7). The fusion process itself provides the particle source: $1 \mathrm{MW}$ of fusion power creates $2 \times 10^{18} \alpha$-particles/s. Under steady-state conditions He is transported to the plasma edge and removed out of the system. The rate of this process is represented by a particle confinement time $\tau_{\mathrm{p}}$. As an empirical fact but also expected from transport theory, $\tau_{\mathrm{p}}$ and $\tau_{\mathrm{E}}$ are linked: $\tau_{\mathrm{p}}$ and $\tau_{\mathrm{E}}$ vary together with confinement regimes and show similar parameter dependences. In fig. $3, \rho=\tau_{\mathrm{p}} / \tau_{\mathrm{E}}$ is assumed constant and varied between 3 and 40. Impurities are released by plasma wall interaction from limiters, divertor target plates and the surrounding walls as preferential impurity sources. The dashed set of curves in fig. 3 shows the impact of carbon radiation on the ignition conditions when the carbon concentration is varied from 3 to $10 \%\left({ }^{5}\right)$. In the ideal case, the ignition conditions require a temperature of about $20 \mathrm{keV}$ and a confinement product $n_{\mathrm{e}} \tau_{\mathrm{E}}$ of $\sim 2 \times 10^{20} \mathrm{~m}^{-3} \mathrm{~s}$. With impurities, the fusion conditions become more critical because of dilution of the fuel and excessive core radiation. Both fuel dilution and radiation lead to an increase in the $n_{\mathrm{e}} \tau_{\mathrm{E}}$ ignition limit. The maximal He-concentration of $50 \%$ translates into a sharp upper temperature limit for ignition, which depends on $\rho$ (see fig. 3). This moves the $n_{\mathrm{e}} \tau_{\mathrm{E}}$ minima toward lower temperatures. Radiation losses cause the temperature minima move to higher values. In this case there are two limits whenever the radiation losses match the $\alpha$-particle source term. Because of the characteristic energy variation of the cross-section (see fig. 2) this happens at two temperatures.

Figure 3 makes it plausible that the He-ash has to be exhausted in a reactor and that fusion plasmas have to be clean, which requires judicious material selection. The technical tool for the exhaust of energy and particles is the divertor, which acts as "ash pan". With divertor the plasma wall contact for exhaust is moved into a separate chamber (see fig. 4) so that material contact close to the plasma surface is avoided.

The curves of fig. 3 are based on carbon $Z=6$ radiation. Carbon is a favoured 1st-wall material in fusion experiments. Because of strong hydrogen absorption it leads to high tritium inventories and has therefore to be discarded. The favoured inner wall material for a fusion reactor is tungsten. The radiation curves of fig. 3 represent tungsten with the impurity concentrations reduced by a factor $\left(Z_{\mathrm{W}} / Z_{\mathrm{C}}\right)^{2} \sim 150$.

There are two ways to satisfy the confinement product $n_{\mathrm{e}} \tau_{\mathrm{E}}$. One is based on large density and low $\tau_{\mathrm{E}}$, the other one on the opposite pairing - large $\tau_{\mathrm{E}}$ and low density. The first case is the goal of inertial confinement fusion research [6]; the second case is the topic of this paper.

$\left(^{5}\right)$ Carbon is used here because the wall of fusion experiments is often fully covered with graphite tiles, a material, which does not melt. $\mathrm{C}$ as a plasma impurity radiates at the plasma edge, which can be tolerated or is even welcome. 


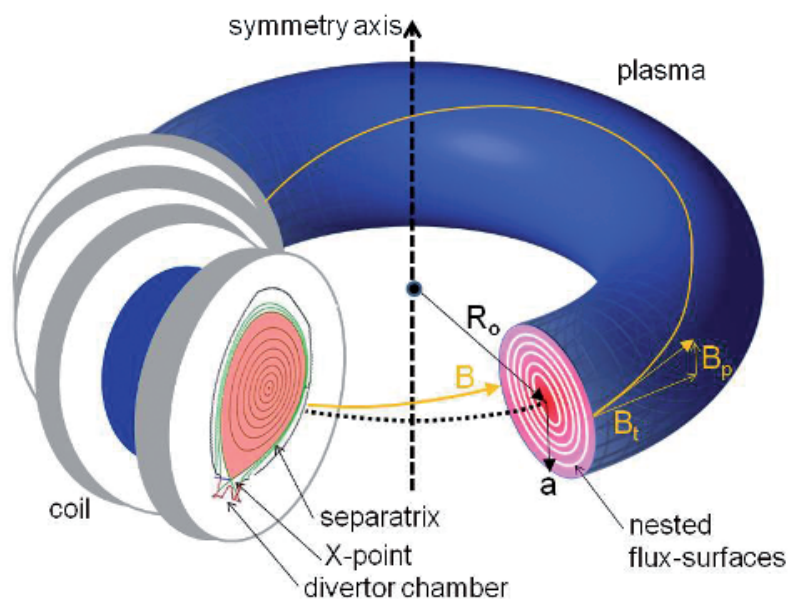

Fig. 4. - Schematic of a toroidal plasma with 4 toroidal coils indicated. Characteristic quantities like the toroidal and poloidal fields, the nested flux surfaces on the right cut, the separatrix of an elongated plasma cross-section with the x-point on the left cut are shown. Also the divertor chamber for exhaust is indicated (left cut).

\section{5. - The tasks of fusion research and the basic features of fusion energy}

The major tasks of fusion $R \& D$ on the basis of magnetic confinement is to confine a hot, high-pressure DT plasma of about $1000 \mathrm{~m}^{3}$ volume by strong superconducting magnets, producing a field of $\sim 10 \mathrm{~T}$. Magnetic confinement has to provide plasma equilibrium and stability and low transport rates. The plasma has to be heated externally by complex techniques to about $20 \mathrm{keV}$ e.g. by the injection of $\sim 100 \mathrm{keV}$ hydrogen particles $\left({ }^{6}\right)$ or by e.m. waves. Under reactor conditions the $\alpha$-particle heating, emerging from fusion processes in the plasma core, takes over and the system enters the phase of a self-sustained burn; the external heating can be turned off. Techniques to remove effectively helium, the ash of the fusion process, have to be developed. Also the plasma has to be kept clean. Impurities would strongly interact with the fuel and contribute with unwanted excess electrons and give rise to enhanced radiation (see eq. (7)). Deuterium will be obtained from sea water by electrolysis. In case of fusion, electrolysis is an inexpensive way to produce deuterium. Tritium, produced in the blanket, has to be stored and distributed in a way to keep the total tritium system inventory small, specifically the fraction of volatile tritium. The blanket is a complex technical component. In addition to its tritium breeding function it has to absorb the neutron heat, about $2-3 \mathrm{MW} / \mathrm{m}^{2}$, and to transfer the power safely to a cooling system, water or gaseous helium. For this purpose, fusion has to develop materials to withstand high heat and neutron fluxes with resistance against material damage. The final production of electricity seems to be the simplest task, which will employ standard steam techniques. The

$\left({ }^{6}\right)$ A plasma of the line density of ITER requires a $\mathrm{D}^{0}$ injection energy of $\sim 1 \mathrm{MeV}$. 


\section{LNES2012}

electrical system efficiency will be in the range of 0.35 to 0.4 . For higher efficiencies, the operational temperatures have to be increased, which has limits because of the complex compound structures used in fusion technology and the loss of material quality toward higher temperatures.

The benefit of this development is a technique, which is capable of delivering large amounts of electricity without serious environmental side effects. The products of the fusion reaction have no environmental relevance, specifically, fusion will not produce $\mathrm{CO}_{2}$. The primary fuels, $D$ with a concentration of $0.016 \%$ in water and $\mathrm{Li}$, are basically inexhaustible and rather homogeneously distributed over the earth, specifically when we consider Li extraction from sea-water $\left({ }^{7}\right)$. Fusion power is intrinsically safe: The fusion process is based on binary collisions and not on an avalanche process, which would necessitate continuous control; the energy density inside the plasma is low; the plasma process stops at the slightest external interference; the involved core energies cannot destroy the containment.

It has to be ensured, however, that tritium is well confined. In [3] the tritium safety problem is discussed in more detail. Another aspect to consider is fusion waste. Though fusion does not produce fissile products like caesium or iodine and it does not produce plutonium or minor actinides, the fusion neutrons activate the minorities inside stainless steel structural material leaving radioactive waste which has to be stored. It is important to note, however, that the time for storage is typically about 100 years and not determined by long decay half-lives. Also this aspect will be discussed further down in more detail.

\section{6. - The basics of magnetic confinement}

Like in the Sun, the conditions in the core of the plasma have to fulfil the fusion conditions as formulated in sect. 4. Under the conditions of magnetic confinement the pressure gradients have to be balanced by the Lorentz force to establish an equilibrium state of force balance. In a magnetic field charged particles move in a helix due to the Lorentz force $\mathbf{F}=q \cdot \mathbf{v} \times \mathbf{B} . q$ is the charge, $\mathbf{v}$ the individual particle velocity, $\mathbf{B}$ the magnetic field. In the direction perpendicular to the field, the excursion of the particle is limited to the Larmor radius $\rho_{\mathrm{L}}$. Because of the specific form of the Lorentz force as vector product of velocity and field, the particle momentum parallel to the field is not changed. Therefore, in a homogeneous field, magnetic confinement is insufficient because it applies to the perpendicular velocity component only. One has to involve and to accept systems with field inhomogeneity to improve confinement. Magnetic confinement systems differ in the way they cope with the confinement parallel to the field thus defining classes of confinement systems.

In linear devices - the first confinement class - parallel confinement is improved utilizing the mirror effect. The basis of the mirror effect is the magnetic moment $\mu=\frac{1}{2} m v_{\perp} / B$ of a magnetised charged particle, which is a constant of motion. An

$\left({ }^{7}\right)$ The economy of this process has not been considered in all details. 
electron or ion, which is thermally agitated with velocity $\mathbf{v}=\left(v_{\perp}, v_{\|}\right)$and which moves from a zone of low field $B_{\text {min }}$ into one with higher field increases its perpendicular velocity $v_{\perp}$ as a consequence of $\mu=$ const. On the other hand, the kinetic energy of the plasma species is also a constant of motion with the corollary that the parallel velocity $v_{\|}$decreases. If the field is sufficiently large $v_{\|} \rightarrow 0$. At this field, the mirror field $B_{\max }$, the particle comes to a full stop. Thereafter, it is accelerated back to the low-field zone by the force $-\mu \cdot \operatorname{grad}_{\|} B$. If the field system is built in a symmetric way, the game repeats itself at the other side with the outcome that the mirror effect causes the particles to bounce between two mirror points and to stay in the neighbourhood of the field minimum thus improving confinement. In an actual mirror machine, characterized by $B_{\min }$ and $B_{\max }$, two classes of particles, discriminated by $v_{\|} / v$, have to be considered. If this velocity ratio is small, a particle is reflected by the magnetic mirrors as described above and represents a trapped one. Otherwise, it is slowed down along its trajectory toward higher field but does not reach the point with $v_{\|} / v=0$. These particles escape the mirror. The boundary is given by $v_{\perp}^{2} / v^{2}=B_{\min } / B_{\max }$ specifying a loss-cone in phase space spanned by $v_{\perp}$ and $v_{\|}$as coordinates.

The confinement of simple mirror machines is insufficient because the transparency of the mirror is too large. Still, we continue describing the confinement situation of the mirror because the concepts help us to understand the physics of more relevant confinement systems. Up to now, we have ignored collisions between the plasma species. Of relevance are the collisions in phase space of the trapped particles occupying the low-field zone scattered into the empty loss cone. Because of their higher velocity, electrons are scattered more frequently into the loss cone than the ions. As a consequence, the plasma is polarised and charges up positively. The formation of an electric field keeps back the escaping electrons by electrostatic means and accelerates the ions. For a transport equilibrium, the so-called ambipolar electric field enforces flux equality $\Gamma_{\mathrm{e}}=\Gamma_{\mathrm{i}}$ between the differently charged plasma species of different masses and different kinetic properties.

A confinement concept avoiding mirror losses is toroidal confinement realised in a plasma ring as shown in fig. 4. In this case, the magnetic coils are arranged in a closed ring thus avoiding end-losses. This geometry defines the class of toroidal confinement systems. An unavoidable feature is the inhomogeneity of the toroidal field with a higher field closer to the vertical symmetry axis. The field gradient points to the symmetry axis. This field inhomogeneity leads to rather unpleasant consequences: The Larmor orbits of the charged particles are not closed causing drifts. Ions and electrons drift parallel to the symmetry axis and leave their magnetic field line. The drift of electrons and ions is in opposite direction leading to an additional vertical electric field. A second parasitic drift appears now in the crossed-field arrangement of vertical electric and toroidal magnetic field. Like in the Hall effect an $\mathbf{E} \times \mathbf{B}$ drift appears perpendicular to both $E$ and $B_{\text {tor }}$ directions, which causes the plasma torus to expand radially. No force equilibrium is established.

The saving idea was to introduce rotational transform. A second field component $B_{\text {pol }}$ is introduced with a perpendicular (poloidal) component. A field line with the components $\left(B_{\text {tor }}, B_{\text {pol }}\right)$ winds around the torus in a helix (see fig. 4 ) and does no longer 


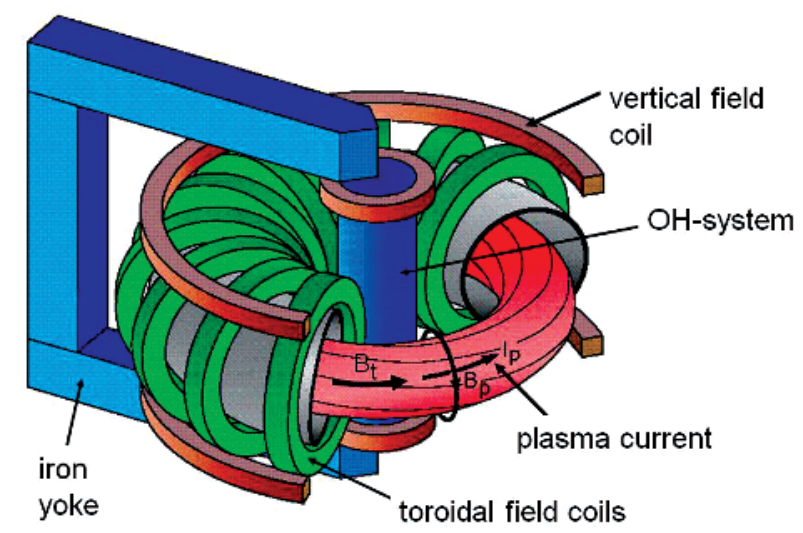

Fig. 5. - Principle set-up of a tokamak with circular cross-section.

stay in a plane rather maps out a toroidal surface. The vertical charge separation is avoided because the up-down sides of the torus are short-circuited by the helical field lines connecting these regions. Macroscopic radial equilibrium can be provided.

The essence of magnetic confinement is the development of a nested system of toroids with field lines, which encircle the tori toroidally and poloidally and with a field line density, which is higher at the inside than the outside (torus effect). The nested toroids, called flux surfaces (see fig. 4), are magnetically isolated and not connected by a field line with a net radial component.

The way the poloidal field component is produced defines confinement classes within toroidal systems. The simplest one is using the potential of a plasma to carry a current. A ring current flowing inside the plasma, the plasma current $I_{\mathrm{p}}$, produces the poloidal field component $B_{\text {pol }}$. As the temperature of the electrons, defining the electrical conductivity of a plasma, is highest in the plasma core, the current density profile $j(r)$ peaks there. Such systems are called tokamaks [7] and are a Russian invention of the '50s of last century $\left({ }^{8}\right)$. Figure 5 shows the principal set-up of a tokamak. The major attraction of the tokamak is that $I_{\mathrm{p}}$ can be produced by a pulsed transformer whereas the plasma ring surrounding a central primary coil acts as secondary winding. In tokamaks, the ratio of $B_{\text {tor }} / B_{\text {pol }}>1$. Another concept with plasma currents is the reversed field pinch (RFP) with $B_{\text {tor }} / B_{\text {pol }} \sim 1$ [8]. Tokamaks and RFPs belong to the category of internal confinement systems because part of the confining magnetic field is produced by currents flowing inside the plasma.

An alternative way is to produce the poloidal field like the toroidal one - by external coils. In this case, the coils have to wind helically around the torus. The field composition reminds of a multipole arrangement with coils being first helically twisted and then bent into a torus. Depending on the current direction inside the helical coils — unipolar or

$\left(^{8}\right)$ The acronym tokamak means: toroidal chamber with magnetic coils. 


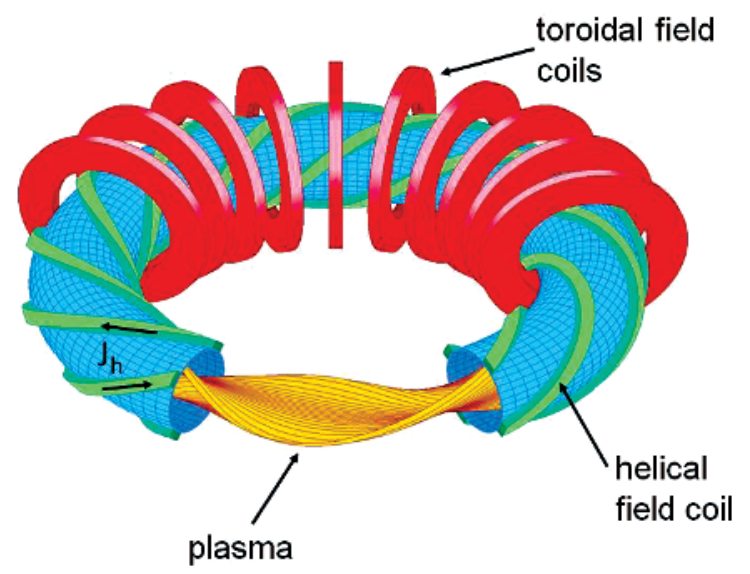

Fig. 6. - Principle set-up of a classical $l=2$ stellarator with helical coils.

bi-polar — we speak of heliotrons/torsatrons or of stellarators. Both belong to the class of helical systems. Stellarators are a US invention of the '50s. Figure 6 shows a sketch of a classical $l=2$ stellarator with helical coils. Helical systems belong to the category of external confinement.

Toroidal systems share common descriptors. In the simplest case with circular poloidal cross-section the torus geometry is defined by major radius $R$ and minor radius a (see fig. 4). The ratio $A=R / a$ is called aspect ratio. The rotational transform $\iota$ of a flux surface is defined by the winding law of the field lines given by $\iota / 2 \pi=A B_{\text {pol }} / B_{\text {tor }}$. RFPs have a larger $\iota$ than tokamaks. In case of helical systems, $B_{\text {pol }}$ is determined in a complex form by the external helical coils system, which is described by the number of coils e.g. 3 heliotron coils with unidirectional current yielding an $l=3$ heliotron. In this case the poloidal cross-section has the shape of a triangle. In case of a stellarator with 4 helical coils and bi-polar currents, we have 2 pairs of coils and an $l=2$ stellarator (see fig. 6). Its poloidal cross-section is elliptical. Another important stellarator descriptor is the coil pitch. The coil winding laws are such that the coils ultimately close. The field pattern of helical systems is periodic in toroidal direction. The toroidal periods can be $m=5$ (Wendelstein 7-A, Germany [3]) or in case of tight windings e.g. $m=10$ (Large Helical Device, LHD, Japan [3]).

Simple tokamaks have a circular cross-section. Equilibria at higher plasma currents are possible when the cross-section is elongated or even has triangular shape. Modern tokamaks benefit from the higher current:

- reaching higher plasma density - a critical ignition and burn parameter (see sect. 4),

- higher stability in terms of the ratio of $\beta=\left\langle p_{\text {kin }}\right\rangle /\left(B^{2} / 2 \mu_{0}\right)$, average kinetic pressure to magnetic field pressure, and

- better confinement, expressed by a larger energy confinement time $\tau_{\mathrm{E}}$. 
We understand the importance of the plasma core in providing conditions for a high fusion yield. Also the surface of the confinement system, the so-called last closed magnetic surface, is of utmost importance. The plasma surface is defined by a separatrix (see fig. 4), which separates (therefore the name) the nested flux surfaces inside from a belt of open field lines at the outside in the so-called scrape-off layer (SOL). The separatrix is formed by a null, an $X$-point, in the poloidal field $B_{\text {pol }}$, which itself is determined by the superposition of the poloidal field of the plasma current with that of additional external poloidal field coils. At the $X$-point (see fig. 4) the field is exclusively the toroidal one. The rotational transform of the separatrix is $\iota=0$. Outside the separatrix, the field lines move around the plasma, are diverted at the $X$-point, separated from the main plasma and finally guided into a separate chamber, the divertor chamber (see fig. 4). This is the basic geometry for exhaust and for the establishment of the plasma wall interaction inside the divertor chamber as also foreseen for ITER and a future fusion reactor. The neutralization of the plasma happens and the transfer of the $\alpha$-particle power (apart from the fraction, which is radiated off at the plasma periphery, the SOL and divertor plasma) occurs onto target plates located inside the divertor chamber. The target plates have to stand high heat fluxes in the range of $15 \mathrm{MW} / \mathrm{m}^{2}$; the divertor chambers are equipped with pumping systems to exhaust He and to control plasma density and composition. Impurities released from the contact zones with the plasma are mostly retained inside the divertor. The magnetics of divertors is still an area of $R \& D$ with new concepts emerging, dubbed Super-X [9] or snow-flake divertor [10].

Also modern stellarators are not simply set-up by coils described by $l$ and $m$. Like in the tokamak case, shaping is essential to improve and optimize plasma performance. The most effective optimization is possible within the concept of multi-helicity stellarators with a mix of poloidal and toroidal $l$ and $m$ parameters. In this case the plasma crosssection varies toroidally changing cross-section according to the dominant local field Fourier coefficients. The benefit of such a configurational complexity is described in sect. $\mathbf{9}^{*}$. It is difficult to realize multi-helicity stellarators by employing helical coils. Instead of separate sets of toroidal and helical coils, the so-called modular coils are used. These are basically individual toroidal coils, which, however, are not planar like in the case of a tokamak rather show specifically shaped lateral excursions (see fig. 12), who line up such that a poloidal field component is generated. Unlike the tokamak, the stellarator does not need additional coils to produce a separatrix. Stellarators are limited by a separatrix as a natural feature of their magnetic system.

Both lines, internal and external confinement, are pursued because of complementary properties. The current of tokamaks represents a strong ohmic heating source for the plasma, $P_{\mathrm{OH}}$, and one can easily reach temperatures around a keV with it. For RFPs the ohmic heating alone may be sufficient to reach reactor conditions. An important advantage of the tokamak is its continuous toroidal symmetry. Hence, the toroidal angular coordinate $\Phi$ is an ignorable one; the consequence is that the associated canonical momentum $p_{\Phi}$ is a conserved quantity. As we will see below, this aspect enters the quality of magnetic confinement.

We have discussed the role of field inhomogeneities in case of the mirror device. 
Toroidal systems also carry a mirror because of the radial field gradient. In a tokamak, there are two classes of particles like in the mirror device. Particles with small $v_{\|} / v$ are affected in their orbit by the increasing magnetic field on their way along the field line toward the inner sector of the torus. If $v_{\|} / v$ is too small the particles are reflected at $B_{\max }$. In this case, they do not freely propagate along the field line and do not experience the full rotational transform, rather they stay at the outside between two mirror points. They are dubbed "trapped particles". Their drift in the field gradient is not fully compensated and they show, therefore, deviations from the flux surfaces much larger than the Larmor radius. Their movement is periodic, however, and they are confined. This is also ensured for the other class of particles. They move freely from the torus outside to the inside and back. They are called free particles. Their deviation from the flux surface is small but modified by the curvature of the toroidal field system. The strength of the axi-symmetric tokamak is that particles move along periodic orbits with finite deviations from the flux surface.

The negative side of the tokamak is the need to inductively produce the current like in a large transformer with the plasma ring representing the secondary winding. This limits the plasma pulses. At the end - maybe even after a few hours - the primary transformer system is exhausted and has to be recharged within minutes. It is not clear yet whether a pulsed system with these features qualifies for a power plant in the $\mathrm{GW}_{\mathrm{el}}$ power range. Therefore, non-inductive current drive techniques are developed using the injection of fast particles or directed e.m. waves. The optimisation of external currentdrive for tokamaks is a dynamic R\&D field.

Other problems emerging from the presence of a strong plasma current are currentdriven instabilities. In a tokamak, the existence of an equilibrium is intimately connected with the existence of the plasma itself and its quality to carry a current. There are nonlinear relations between equilibrium, transport and stability properties, which allow the development of instabilities causing degradation up to the limit of self-destruction of the plasma in a disruption. In case of a reactor, $20 \mathrm{MA}$ or so may thus be switched-off within a fraction of a second. The electro-mechanical dynamics of such a perturbation leads to high voltages or high currents, $I_{\text {ind }}$, induced in structural components leading to strong $I_{\text {ind }} \times B$ forces which have to be safely handled.

An interesting aspect of the RFP is that the current is maintained self-consistently by a dynamo process inside the plasma. Strong magnetic turbulence adds up in a coherent way to produce a plasma flow with velocity $v_{\text {dyn }}$, which induces an electric field $E_{\mathrm{dyn}}=v_{\mathrm{dyn}} \times B$, which itself drives poloidal current giving rise to most of the core toroidal magnetic field. The catch of this simple concept is the lack of nested flux surfaces being replaced by a rather chaotic field pattern caused by a high magnetic turbulence level allowing only low confinement. High transport levels are the major concern for RFPs. Though the programmatic validity of the RFP might be questioned, its plasma has rich physics resembling in some aspects those observed in stellar plasmas allowing to study astrophysics phenomena in the laboratory. It has also interesting aspects of self-organisation of thermodynamically open systems, which we will present in sect. 7 . 
The plasma geometry of stellarators is invariably 3-dimensional. No equilibrium can be constructed with continuous symmetry in the current-free case when $\operatorname{rot} \mathbf{B}=0$. In addition to the toroidal curvature effect helical systems show a further field inhomogeneity, which is introduced by the helical coils (or by the aligned lateral coil excursions of modular coils). The field beneath the helical coils is larger than the one between these coils. As a consequence, there is a third class of particles complementing the free and toroidally trapped ones - the helically trapped particles. These particles are highly localized in phase space, benefit only little from the confining effect of rotational transform with the consequence of a net radial drift out of the system. They lead to intolerable losses under reactor conditions.

A more fundamental treatment of particle confinement starts from the 3-dimensional field geometry of a helical system, the lack of continuous symmetry replaced by discrete toroidal periodicity. In such a case there is no toroidal (tokamak, RFP), poloidal (mirror device) or helical (linear stellarator) symmetry established as in principle possible for magnetic confinement. The corollary of the lack of symmetry is that no component of the canonical angular momentum is conserved and that collisionless losses in phase space occur. As this "loss-cone" is continuously filled by collisions, helical systems have difficulties to meet the ignition and burn conditions unless the problem with the lack of symmetry is removed. This is the story of the so-called quasi-symmetric stellarators as described in sect. $\mathbf{9}^{*} 2$.

\section{7. - Characteristics of high-temperature fusion plasmas}

A fusion plasma has to fulfil many requirements - equilibrium, stability, good confinement, plasma purity, and other qualities. This requires a rigorous strategy of optimisation. It involves high-level physics issues like the understanding of turbulent plasma transport and its "taming", but also technical issues like the qualification of compound structures able to withstand the contact with the plasma. Here we will limit ourselves to only discussing one aspect, plasma transport and resulting confinement, as it is the most critical issue and possibly the most interesting one from the scientific point of view. Reference [11] represents a comprehensive review of the physics of magnetic confinement.

Plasma transport has two branches - a laminar one and a turbulent one. The laminar processes are based on Coulomb collisions between charged species, which cause particles to jump from one flux surface to another one and - according to the plasma gradients from the inside to the outside as net effect. In a homogeneous plasma, the characteristic step-parameters of this diffusive process are the Larmor radius $\rho_{\mathrm{L}}$ and the Coulomb collision time $\tau_{\mathrm{Cb}}$. In toroidal geometry these two parameters are modified because of the induced drifts. Of relevance are the trapped particles because of their large step size of $\sim \rho_{\mathrm{L}} B_{\text {tor }} / B_{\text {pol }}$, the Larmor radius in the poloidal field. The effective collision time is determined from the collisional flow of particles across the boundary in phase space separating free and trapped particles. The laminar transport processes, which are described by a complete theoretical edifice, the theory of neo-classical transport [12], do not play a major role for the tokamak losses. This is different in stellarators. Under 
fusion relevant conditions the collisionless losses of helically trapped particles become dominant. Like in the case of mirror devices, their losses are not intrinsically ambipolar causing a radial electric field, which itself affects particle orbits, specifically those of ions. The transport equation becomes non-linear: the electric field reduces the losses but leads also to different confinement branches featuring widely different plasma transport characteristics.

Bifurcations in laminar transport can happen under 3-D conditions but more so with turbulent transport irrespective of the system symmetry. Like the Sun, also technical plasmas are highly turbulent [13]. Thermodynamically, plasmas represent open systems characterized by non-linear relations, which also govern turbulent transport. Pressure gradients and plasma currents represent the free energy, which drive plasma turbulence. There are de-stabilizing properties like plasma resistivity, or geometrical aspects like the collinearity of pressure and field gradients at the outer half-sector of the torus, or socalled rational flux surfaces where a field line does not ergodically map out the flux surface rather closes upon itself after a number of toroidal and poloidal circulations. Resistivity decouples the conducting medium (the plasma) from the magnetic field; the diamagnetic nature of the magnetized plasma causes it to move outward into areas of low magnetic field. This tendency is supported by the pressure gradient at the torus outside (and opposed by the one at the inside). In the third case the topology of a global instability as an eigenmode of the bound geometry coincides with the topology of the rational field line closing upon itself. Under these circumstances a mode or instability does not have to invest energy for the distortion of the field topology and can grow with less prohibition.

The transport relevant turbulence scales with the Larmor radius. The non-linear relations between stabilizing and de-stabilizing contributions give rise to onset conditions and define critical gradients for temperature and pressure. If, e.g., the temperature gradient grows beyond a critical threshold value, the level of turbulence rises strongly. Formally, the diffusivities depend themselves on the driving force gradient. Therefore, gradients are clamped to the critical ones.

Here we can understand why fusion requires the size already of a reactor to demonstrate its scientific feasibility. In order to meet the high temperature of $20 \mathrm{keV}$ in the plasma core ensuring a high fusion yield, steep gradients are necessary. Those are, however, governed by critical onset-conditions and limited to critical values. The situation is reminiscent of a sand pile. In the effort to increase its height, critical gradients send excess sand in little avalanches to the base of the pile. Only by broadening the base, the peak can be raised obeying the overriding critical condition. In principle, the same is observed in fusion plasmas. In order to meet the core conditions, the volume has to be increased leaving fixed the normalized temperature gradients $\nabla T / T$. Thus, the plasma volume increases and more fusion power is produced with the upshot that fusion can only be demonstrated at the power level of a few $100 \mathrm{MW}$.

Important is therefore to overcome the conditions leading to profile similarity and to improve confinement. This would allow realising compact and economic systems. Here, a characteristic feature of plasmas comes to rescue, which emerges from its nature as open system, operated far from thermodynamic equilibrium viz. to self-organise. We 

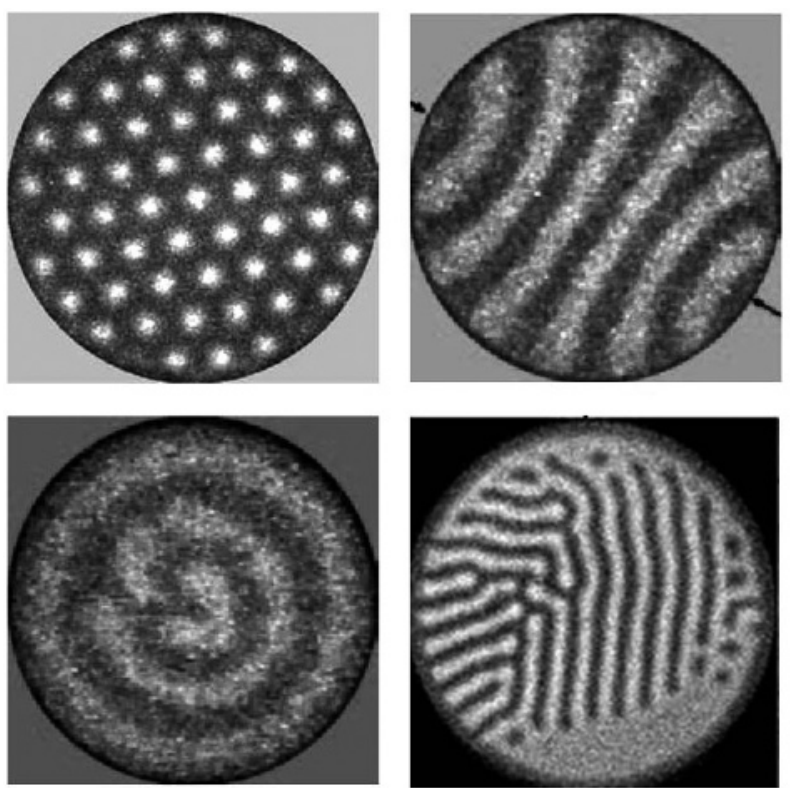

Research Group Purwins, UNI Münster, 2005

Fig. 7. - Examples of current density distributions of dielectrically suppressed diode discharges. Starting form top/left, a hexagonal pattern, a pattern with periodic stripes, a rotating spiral pattern and a case with bright filaments is shown. These data are obtained by the Research Group Purwins of the UNI Münster [12] (courtesy, H. Purwins).

demonstrate self-organisation in space first by a most simple plasma arrangement - a diode, which is operated with an ac-voltage. In order to prevent the formation of an arc, the circular electrodes are protected by di-electric layers. This arrangement allows quasitwo-dimensional dielectric-barrier discharges. The ac-current density does not develop homogeneously across the electrodes rather organizes itself in patterns, which depend on the diode current as control parameter. Figure 7 shows examples of such patterns [14]. This phenomenon is not unique to plasmas rather a universal phenomenon described by reaction-diffusion theory in the frame of non-equilibrium thermodynamics [15].

An interesting spontaneous transition into a state with better confinement is observed in RFPs. Under normal conditions strong multi-helicity magnetic turbulence governs transport and confinement. The turbulence level is large enough to ergodize the magnetic field within the confinement zone. If the system is driven hard, e.g. by increasing the plasma current, it suddenly transits into a regime with a lower turbulence level. Spontaneously, in the core, the ergodized field is replaced by nested flux surfaces adopting a quasi-single-helicity equilibrium. Figure 8, left, exemplifies such a case. The temperature in this zone shows a distinct increase. The plasma self-heals a defect improving confinement thereby. Figure 8, right, shows this configurational change with an island in the poloidal cross-section containing nested flux-surfaces giving rise to the benefit of a distinct local increase in temperature [16]. 

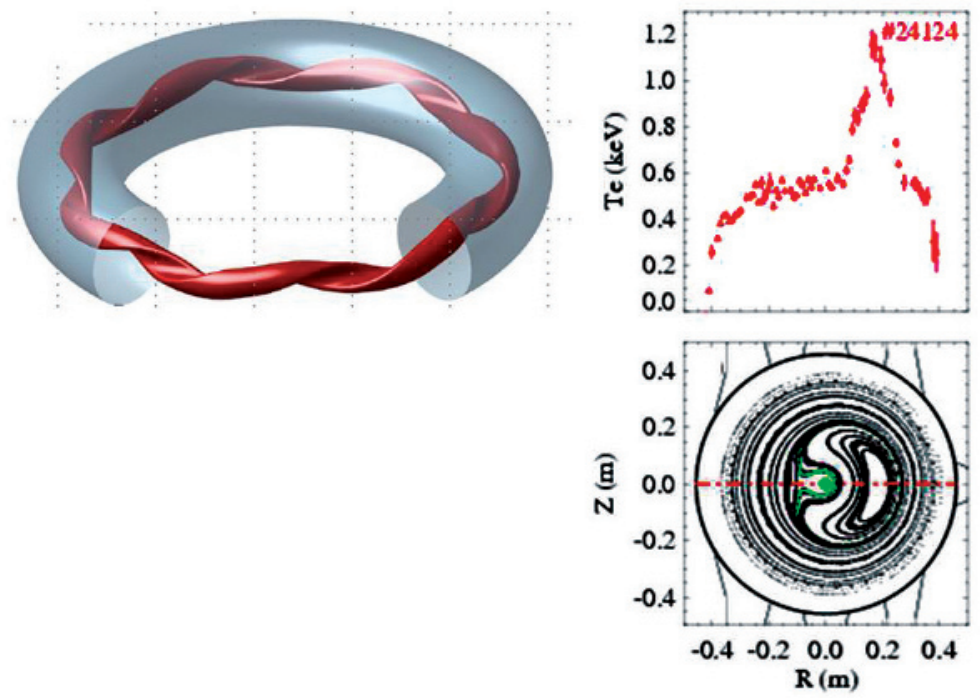

Fig. 8. - Left: The development of a single-helicity state in the plasma core. Top right: electron temperature with a peak in the radial range of the helical equilibrium. Bottom right: poloidal cross-section with nested flux surfaces and chaotic field lines closer to the edge (courtsey, Consortia RFX).

Also in tokamaks and stellarators, bifurcations into regimes with higher confinement are observed, which are of programmatic relevance and allow realizing the fusion conditions under simpler and more realistic conditions. Figure 9 shows the transition from the L-regime, the low confinement regime, to the H-mode, its high-confinement counterpart [17]. The example is taken from ASDEX Upgrade tokamak. Also in this case, the transition is a threshold process, which requires a stronger drive developing after surpassing a heating power threshold. The transition occurs as soon as NBI heating has been added (see fig. 9a). The 2-dimensional turbulence at the plasma edge changes its dynamic behaviour (see fig. 9b) whereas the spectral content condenses toward large scales limited only by the minor plasma radius: the interaction of turbulent eddies sum up to a coherent poloidal flow field (see fig. 9c). The sheared flow now acts back onto its driving agent and tears apart — de-correlates - the turbulent eddies. The turbulence is strongly reduced and steep gradients develop at the plasma edge forming - what is called - a transport barrier. When the turbulence is suppressed, the steep gradients for their part are maintained by equilibrium conditions where the steep ion pressure gradient is balanced by a negative radial electric field, which itself is equivalent to a strong and sheared poloidal flow. The state of low-turbulence is preserved. The turbulence quench acts as a trigger mechanism to a state with low turbulence and improved confinement. The prospects and expectations of ITER are based on H-mode confinement qualities. The generation of flows in the interaction with $2 \mathrm{D}$ turbulence is reminiscent of the mechanisms, which give rise to the belts of the planets Jupiter and Saturn and which play a role in atmospheric and oceanic flows. 


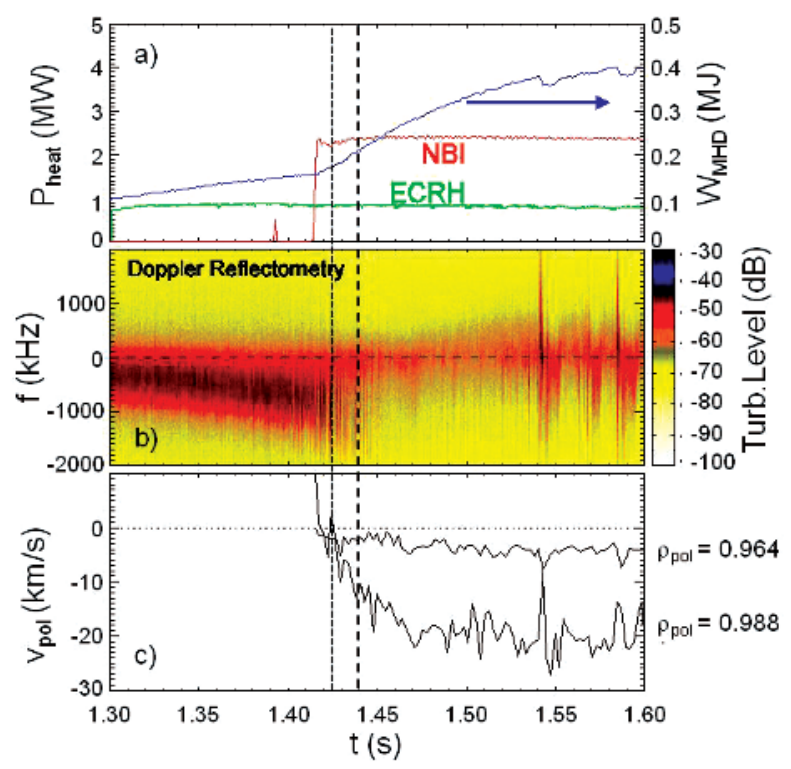

Fig. 9. - Different plasma traces from ASDEX Upgrade tokamak in a time window from 1.3 to $1.6 \mathrm{sec}$ during a discharge with auxiliary heating (NBI: beam heating; ECRH: electron cyclotron heating) with an $\mathrm{H}$-transition (vertical lines). At about $1.425 \mathrm{~s}$, the discharge transits into a period with limit-cycle oscillations; the final H-transition occurs at $1.44 \mathrm{~s}$ (dashed line). a) Plots the energy content together with the power of the two heating methods; b) plots frequency and amplitude (colour coding; log. scale) of the turbulence; c) shows the poloidal plasma rotation for two normalised radii $(\rho=r / a)$. Poloidal rotation increases strongly at the plasma edge, about $1 \mathrm{~cm}$ inside the separatrix.

The potential of self-organisation is largest in RFPs because both confining fields are generated in a self-consistent manner. The stellarator is the opposite, because both fields are produced from the outside under full control. There is an interesting parallelism between the development of the QSH-state of RFPs and the H-mode. Both systems have to be driven hard from the outside. The free energy has to be increased in one case by the current, in the other case by the plasma pressure. In both cases turbulence is reduced in selected regions - in the core or at the edge, respectively. In both cases, the coherent interaction of turbulence gives rise to plasma flows. A transient process leads to a new equilibrium situation where the essential electric field is maintained either by the helical plasma deformation or the increased ion pressure.

\section{8. - Status of fusion energy development}

Fusion research was started worldwide after world-war II. The EU fusion programme is jointly coordinated by the Euratom contract, which provided the legal basis and the means to build the presently largest and most relevant fusion device, the Joint European Torus, JET, a tokamak, operated in UK close to Oxford. This device is closest to the 
ignition conditions. In the last decade, the Asian fusion programme has made impressive progress with modern tokamaks in Korea (KSTAR [18]), China (EAST [19]), India (SST$1[20])$. Japan has a long tradition in fusion development and houses the largest helical system at present, LHD, a heliotron [21]. Japan is now constructing a large tokamak, JT-60 SA [22]. All Asian tokamaks are realised with superconducting coils and address the critical and not yet solved problem of steady-state operation, specifically critical for tokamaks owing to their intrinsically pulsed nature. Helical systems are operated in Australia, Germany, Japan, Spain, Ukraine, and the USA. RFPs are operated at many places at the scale of university experiments. The largest devices operate in Italy (RFX [23]) and USA (MST [24]).

In separate experiments, fusion has already achieved the necessary temperature and density. Temperatures of $40 \mathrm{keV}$ can be produced in a routine fashion basically by pressing a knob. Densities have been achieved a factor of $\sim 10$ above the required one. The critical parameter is $\tau_{\mathrm{E}}$, where a factor of 4 is missing for reaching ignition. It is a major effort of the world-wide fusion programme to improve confinement quality beyond the one achieved with the H-mode. Recently, it was shown that transport barriers, as observed in the H-mode at the edge, can also be produced additionally in the core region in the form of internal transport barriers [25]. Intense fusion research still pays off.

The US experiment TFTR and JET have carried out DT experiments without any technical problems. Of course, the tritium consumption is in the range of grams and not kilograms as necessary in case of a reactor. $\alpha$-particle heating has been demonstrated. Most importantly, JET has demonstrated in a short pulse a fusion power of 16.1 MW in DT experiments in the $\mathrm{H}$-mode equivalent to a power amplification factor $Q=P_{\text {fus }} / P_{\text {ext }} \sim 0.65$.

The fusion community was able to support unanimously one concept for the first fusion test reactor, ITER. This device is based on the accumulated word-wide knowledge with a strongly shaped tokamak plasma incorporating a divertor for exhaust. The first-wall materials are in agreement with the best knowledge available at present.

The international efforts of fusion research are best demonstrated by the joint data base, which provides the scaling of the energy confinement time $\tau_{\mathrm{E}}$ defining the operation point of ITER. As $\tau_{\mathrm{E}}$ is determined by turbulent processes a precise prediction of the ITER $\tau_{\mathrm{E}}$ value is still not possible though the basic mechanisms are understood and the equations and the formal structure for turbulence modelling developed. Its multiparameter dependence has to be identified by many experimental results subject to a rigorous statistical regression analysis. The outcome of such a study is shown in fig. 10 . The experimental $\tau_{\mathrm{E}}$ value depends on many parameters, plasma current $I_{\mathrm{p}}$, toroidal field $B_{\text {tor }}$, heating power $P$, density $n$, isotopic mass $M$ and the key geometry parameters, major radius, $R$, elongation, $\varepsilon$, and triangularity of the plasma cross-section, $\kappa$ :

$\tau_{\mathrm{E}}^{\mathrm{ITER}}(\mathrm{s})=0.0365 I_{\mathrm{p}}{ }^{0.97}(\mathrm{MA}) B^{0.08}(T) P^{-0.63}(\mathrm{MW}) n^{0.41}\left(10^{20} \mathrm{~m}^{-3}\right) M^{0.20} R^{1.93}(\mathrm{~m}) \varepsilon^{0.23} \kappa^{0.67}$.

The expected $\tau_{\mathrm{E}}$ value of ITER is also shown $(3.7 \mathrm{~s})$. It is in agreement with the objectives of ITER and defines several crucial parameters of its design. 


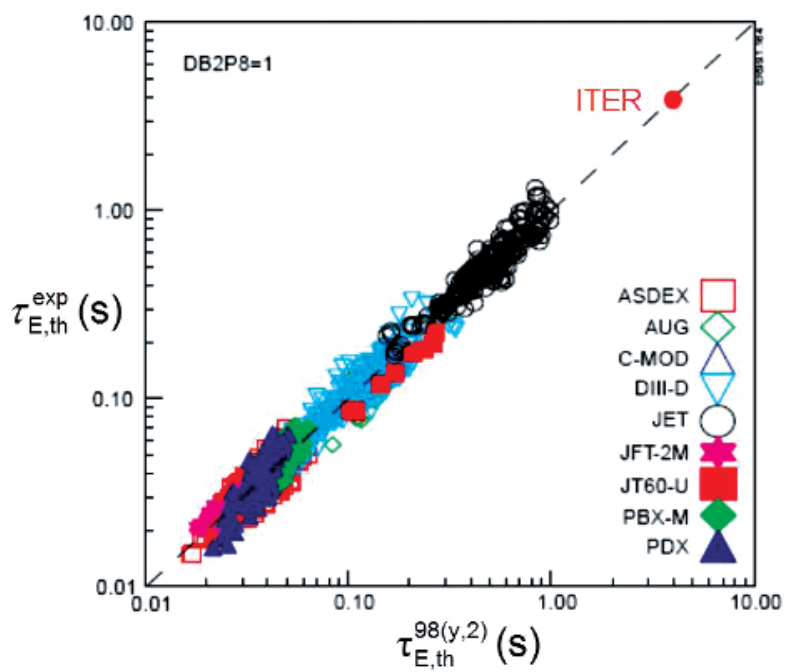

Fig. 10. - Multi-machine thermal energy confinement time $\tau_{\mathrm{E}, \mathrm{th}}{ }^{\exp }$ against the scaling results of the 98(y.2) ITER scaling. The expected ITER confinement time is also shown.

Stellarators can operate at higher density than tokamaks, which is an advantage for high-power divertor operation. They show similar parameters as tokamaks of similar size and field. They do, however, not reach the high temperatures and confinement times as the large tokamaks because of the smaller scales of the present-day helical systems.

\section{9. - The next development steps}

9'1. ITER. - ITER, shown as a computer drawing in fig. 11, will be the first fusion reactor producing fusion energy aiming at a power gain $Q=10$. ITER has major physics issues to explore and to clarify, which necessitate a burning plasma state. The most important ones are (1) the confinement characteristics with dominant self-heating, (2) burn control and helium-exhaust, and (3) plasma stability in the presence of a fast ion component, the $\alpha$-particles. Crucial are also the properties of helium transport from the core to the edge and the safe removal of He within the divertor. Only a helium concentration of $2 \%$ is tolerable in the reactive core (see fig. 3). The He-fluxes have a convective and a diffusive component. It is expected, but not ensured that the convective part will move He ions to the outside. The $\alpha$-particle pressure also represents a contribution to the free energy with the potential to drive instabilities. In this case, Alfven waves could be induced by a velocity resonance within the spectrum of slowingdown $\alpha$-particles, which could act back onto the driving mechanism and reduce the confinement of energetic particles needed for plasma heating. Another crucial area of exploration with ITER is external current drive to prepare the conditions for steady-state tokamak operation. This effort combines suitable techniques with judiciously tailored 


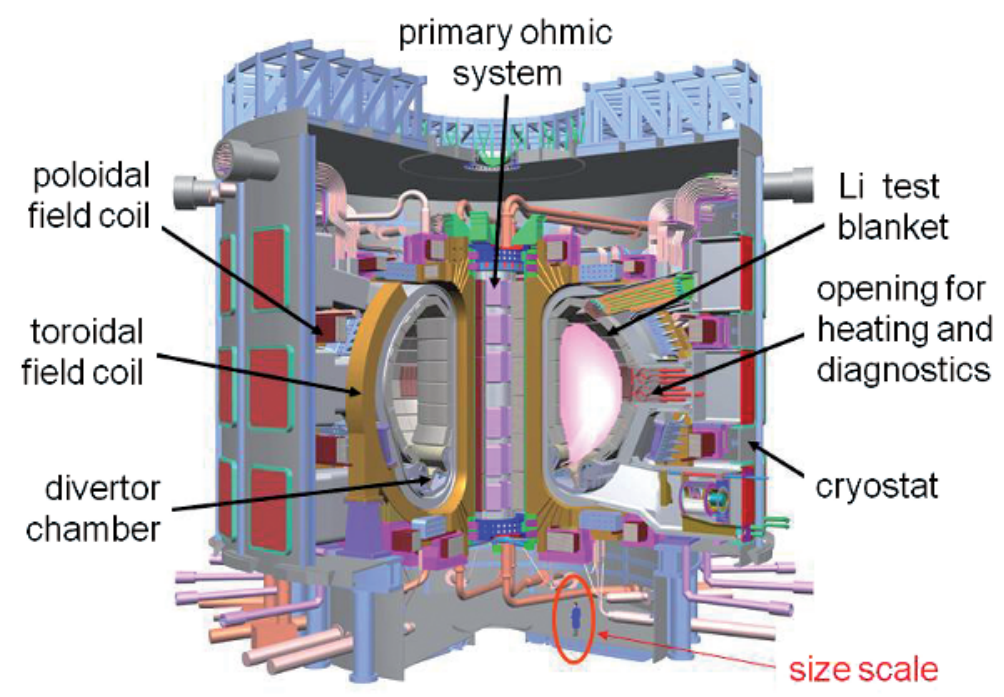

Fig. 11. - Computer drawing of ITER.

plasmas, which maximise internal currents (bootstrap current) minimizing thus the needs for external contributions.

There is a large field of technological contributions expected from ITER. As only real projects solve practical problems and provide the necessary technologies, ITER plays a unique role in fusion technology also. ITER will demonstrate the use of superconductivity in the harsh environment of fusion, the viability of remote handling techniques for the complex assembly and maintenance tasks inside the plasma vessel and it will develop ambitious external heating and current drive concepts like the injection of high-energy hydrogen atoms at $1 \mathrm{MeV}$ energy and in the $100 \mathrm{MW}$ power range. Critical issues are the exhausted $\alpha$-power handling at a level of $15 \mathrm{MW} / \mathrm{m}^{2}$ and the demonstration of tritium production using test blankets in a form, which allows clear modelling predictions for those used in DEMO and a later power reactor.

9.2. Wendelstein 7-X and the HELIAS reactor. - As we have seen, stellarators need confinement optimisation. The most rigorous form led to the concept, which underlies the Wendelstein 7-X (W7-X) project to be realised in Greifswald, Germany, which is presently (2012) in the assembly phase (see fig. 12). The theoretical background of the optimisation is (1) that the deviation of particles from the flux surface as well as equilibrium and stability properties depend on the variation of the magnetic field strength $|B|$ in the flux surface [26] and (2) that $|B|$ can be made 2-dimensional in an otherwise 3 -dimensional toroidal geometry [27] as it is given fact for external confinement. Systems with these properties are called quasi-symmetric systems and meanwhile several smaller stellarators are built and operated based on these concepts (HSX in Wisconsin [28], Heliotron J in Kyoto [29]). The design of W7-X follows the quasi-isodynamic principle. 


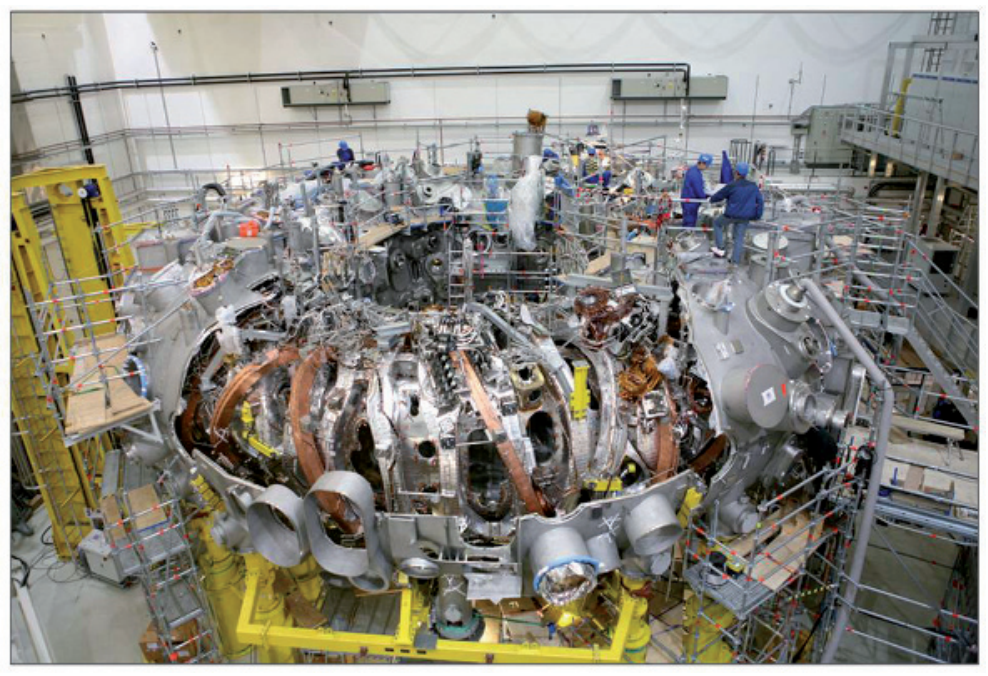

Fig. 12. - Photo into the experimental hall of Wendelstein 7-X (status Dec. 2011).

In an isodynamic system [30] all flows of particles, heat and charges would ideally occur in a flux surface without radial component. Such a system cannot be realised in the frame of toroidal confinement. Quasi-isodynamicity, a viable approximation, foresees rigid equilibrium and stability toward high plasma pressure and good confinement for thermal and energetic particles ( $\alpha$-particles) avoiding the helical ripple losses as described in sect. 6. One optimisation feature is the utilisation of the mirror effect. The toroidal periodicity of W7-X corresponds to a pentagon. Straight sectors are connected by corners. The toroidal curvature is therefore localised to the corners. In order to minimise the drift losses in the zones of field inhomogeneity, the field strength $|B|$ is increased in the corners. W7-X resembles a system of linked mirrors. The most critical particles to drift off the system, those with a large $v_{\perp} / v$, are expelled from the sectors with curvature. The complete and rigorous optimisation is still not verified. Some of the basic physics qualities have, however, already been tested in the smaller and partially optimised stellarator device W7-AS [31]. These favourable properties - if they become reality - are on top of the intrinsic stellarator characteristics — steady-state operation free of current-driven instabilities. W 7-X, being a superconducting device, is supposed to demonstrate these features for pulses up to $30 \mathrm{~min}$.

Stellarator reactor studies have the main emphasis on stellarator-specific aspects such as the coil system and the complex 3D geometry of blanket and neutron shield. They are basically extrapolations of the present concepts, in case of W7-X it is the HELIAS reactor concept [32]. The design is dictated by two criteria: at first the size must be large enough to accommodate blanket and shield between plasma and coils and secondly the confinement time, which roughly grows with the plasma volume, must be large enough to ensure ignition and self-sustained burn. Not explored in much details yet are the 
necessary properties subsumed under the term RAMI — reliability, availability, maintainability and inspectability. The 3D nature of stellarators will complicate the technical realisation of the RAMI criteria. Otherwise, stellarators have to demonstrate the same qualities as foreseen for ITER and described in the previous chapter. Of course, also stellarators will benefit tremendously from the additional physics and the technology provided by ITER. Whether an intermediate step will be necessary in the stellarator reactor evolution like a "stellarator ITER" is probable but needs to be seen.

A future stellarator reactor will not need a current drive system. About $200 \mathrm{MW}$ current drive power are required for a steady-state tokamak reactor. The plant electricity consumption and the recycling power are large for a typical electrical efficiency of 0.4 . This could increase the cost of electricity by $25 \%$ [33].

9.3. DEMO. - The DEMO will be an electricity producing fusion reactor prototype, which will deliver about $1 \mathrm{GW}$ electric power steady-state or quasi-steady state. It will be slightly larger than ITER. Specifically, it will operate at higher density $\left(P_{\text {fus }}\right.$, being based on a binary process, scales with $n^{2}$ ) and will require somewhat higher confinement and stability margins. These topics are on the agenda of the ITER research programme. ITER will also play an important role for DEMO in the field of licensing. This is a totally new area, which will be exercised for the first time with the nuclear safety authorities of France.

The DEMO could be a tokamak or a stellarator. It could also be that the global energy demand is so critical in one or two decades from now that more means are provided than is presently the case for energy research so that fusion $R \& D$ could continue on a much broader basis.

\section{REFERENCES}

[1] Garry McCracken and Peter Stott, Fusion: The Energy of the Universe (Academic Press) 2nd edition; ISBN-13: 978-0123846563.

[2] Kikuchi M., Lackner K. and Tran M. Q. (Editors), Fusion Physics (IAEA) 2012, ISBN 9789201304100.

[3] Wagner F., IPP-report 18/03.

[4] Lawson J. D., Proc. Phys. Soc. B, 70 (1957) 6.

[5] "Progress in the ITER Physics Basis", Nucl. Fusion, 47 (2007) 1-414.

[6] Atzeni S. and Meyer-ter-Venn J., The Physics of Inertial Fusion (Oxford Science Publication) 2004, ISBN 01985622640.

[7] Wesson J., Tokamaks (Oxford University Press) ISBN 9780198509227.

[8] Bodin H. A. B. and Newton A. A., Nucl. Fusion, 20 (1980) 1255.

[9] Valanju P. M. et al., Phys. Plasmas, 16 (2009) 056110.

[10] Ryutov D. D. et al., Phys. Plasmas, 15 (2008) 092501.

[11] Boozer A. H., Rev. Mod. Phys, 76 (2005) 1071.

[12] Hazeltine R. D. and Meiss J. D., Plasma Confinement in Books on Physics Series (Dover) 2003, ISBN 9780486432427.

[13] Yoshizawa A., Iтон S. I. and Ітон K., Plasma and Fluid Turbulence: Theory and Modelling (Taylor\&Francis) 2002, ISBN 9780750308717. 


\section{LNES2012}

[14] Purwins H.-G., IEEE Trans. Plasma Sci., 39 (2011) 2112-2113; Special Issue on "Images in Plasma Science".

[15] Grindrod P., The Theory and Applications of Reaction Diffusion Equations: Patterns and Waves, in Applied Mathematics and Computing Sciences Series (Oxford) 1996, ISBN 978018500049.

[16] Martin P. et al., Plasma Phys. Contr. Fusion, 49 (2007) A177.

[17] Wagner F., Plasma Phys. Contr. Fusion, 49 (2007) B1.

[18] LeE G. S. et al., Nucl. Fusion, 41 (2001) 1515.

[19] Wu S. et al., Fusion Engin. Design, 82 (2007) 463.

[20] Saxena Y. C. et al., Nucl. Fusion, 40 (2000) 1069.

[21] Iryoshi A. et al., Nucl. Fusion, 39 (1999) 1245.

[22] IsHidA S. et al., Nucl. Fusion, 51 (2011) 094018.

[23] Rostagni G., Fusion Engin. Design, 4 (1995) 301.

[24] Prager S. C. et al., Phys. Fluids B, 2 (1990) 1367.

[25] Wolf R. C., Plasma Phys. Contr. Fusion, 45 (2003) R1.

[26] Boozer A. H., Phys. Fluids, 23 (1980) 904.

[27] Nührenberg J. and Zille R., Phys. Lett. A, 129 (1988) 113.

[28] Anderson F. S. B. et al., Fusion Technol., 27 (1995) 273.

[29] OBiki T. et al., Nucl. Fusion, 41 (2001) 833.

[30] Palumbo D., Nuovo Cimento B, 53 (1968) 507.

[31] Hirsch M. et al., Plasma Phys. Contr. Fusion, 50 (2008) 053001.

[32] Igitkhanov Yu. et al., Fusion Engin. Design, 81 (2006) 2695.

[33] Ward D., Fusion Sci. Technol., 56 (2009) 581. 